\title{
Gate Drives generating EMI in the absence of Power Converter loading
}

\author{
Arnoldus S. de Beer, Member, IEEE
}

\begin{abstract}
This paper shows how a power converter can generate electromagnetic interference (EMI) through the operation of gate drives only - in the absence of any power conversion. This is due to parasitic capacitances connecting the power semiconductor's gate to the main circuit. A half bridge converter is used to illustrate this concept. Practical measurements are compared to simulations for an energized and non-energized converter. Even without loading, a converter can exceed regulatory conducted emission EMI limits. This effect is important to consider during the design of converter EMI mitigation - especially for low power converters where the load current is not dominant.
\end{abstract}

Index Terms-Electromagnetic Interference, Conducted EMI, Gate drives, Power Convertors, Power electronics, Power semiconductor switches, Switching converters

\section{INTRODUCTION}

$\mathrm{P}$ OWER convertors play an important role in the generation and distribution of electricity. This importance will increase as the world moves towards the Smart Grid and the Internet of Things (IoT). The Smart Grid will optimize electrical energy usage[1] and the IoT will increase connectivity [2]. With this move it is envisaged that power converters will be ever more

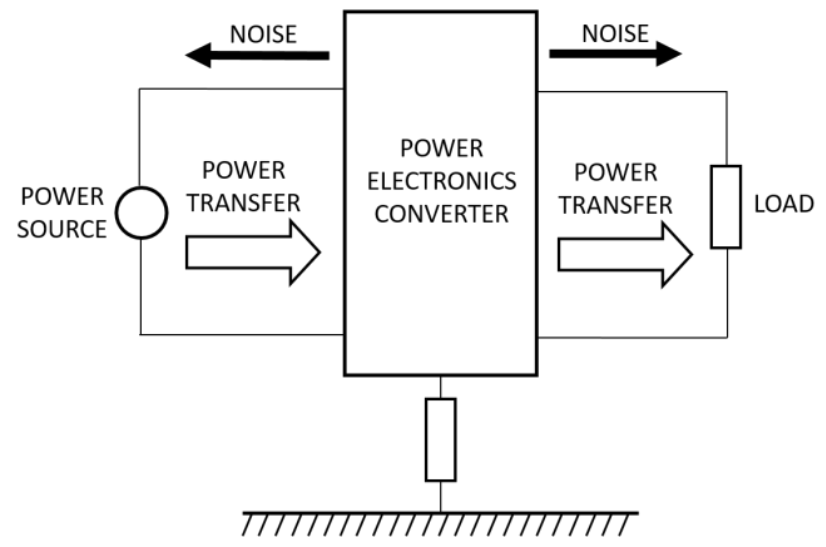

Fig. 2. Direction of noise flow in a switching power converter as opposed to the main (converted) energy flow. Engineering, University of Johannesburg, Johannesburg, South Africa. (e-mail: asdebeer@uj.ac.za).

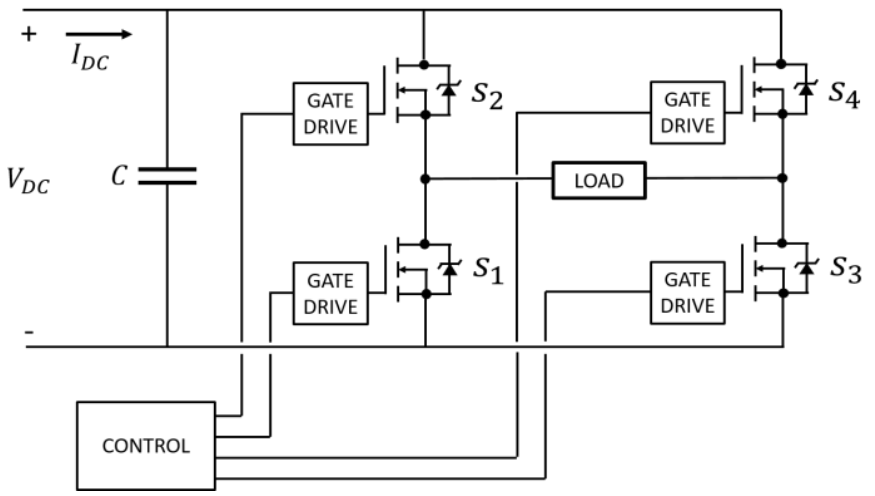

Fig. 1. Example of a power electronic converter $\mathrm{H}$-bridge with the semiconductor switches (MOSFETs) driven by Gate Drives.

present and closer to each other. Electromagnetic Interference (EMI) can inhibit safe operation of converters [3] as they can mutually interfere due to conductive or radiative coupling in close proximity.

Converters generate electrical noise (or interference) due to a switching action. Although some converters are nonswitching or linear, power converters are predominately switching as switching reduces losses during the conversion process. It is also necessary so that voltage levels can be scaled.

It is important however to conceptually distinguish between the main energy flow (or converted energy) in a converter as opposed to the flow of noise. This is graphically shown in Fig. 2. Although a matter of convention, energy flow to the converter should be seen as to emanate from a source, being converted and flowing towards the load. Noise on the other hand is produced in the converter, and flows on the supply line towards the source. At the output of the converter, noise flows towards the load. It is the noise flowing on the supply line that can interfere with other equipment on the same network. To keep this type of conducted interference from happening, most electronic products with switch mode power supplies have a filter on the supply lines to keep the noise from reaching the supply.

Switch mode converters use semiconductor devices such as IGBTs (Insulated Gate Bipolar Transistors), Thyristors and 


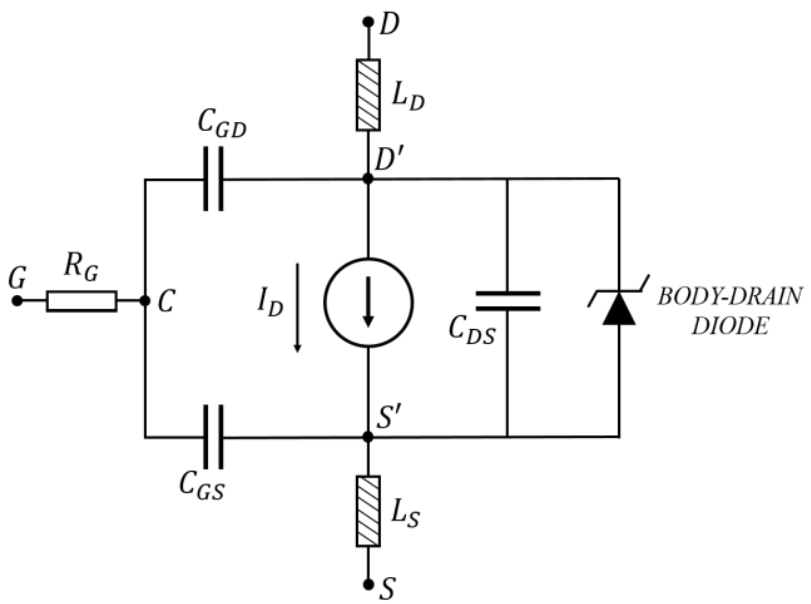

Fig. 3. Model of a MOSFET used to model switching behavior.

MOSFETs (Metal Oxide Semiconductor Field Effect Transistors) as switches. These switches are controlled by digital logic and modulated by software to produce an output. Between the controller and the power switches are circuits that drive the gates of the semiconductor switches. These are called gate drivers. This is shown in Fig. 1. load.

In Fig. 1, switching noise will be present at $I_{D C}$ as well as the

It is common practice when designing power line filters to consider the converter switching action at maximum load (maximum $I_{D C}$ ) for sizing the filter [4]. The assumption is that all the noise from the converter is due to the converter energy transformation process. Following this line of reasoning it can be assumed that if the converter has zero output the interference will also be zero. It is shown that this is not the case.

The contribution of this study is to show that even if zero electrical energy is converted, gate drive action still produces considerable noise on the power lines. This is due to the fact that there are parasitic components (mainly capacitance) between the gates of power switches and their connections to the DC rail (busbar) [5].

In this study MOSFETs are used as an example of a power electronic switch. Background is given showing that MOSFETs have parasitic capacitances. It is shown how these capacitances can couple noise components onto the busbars.

The next sections of this study show results obtained by simulation and practical measurements. For both the simulation and measurements conducted, EMI on the power line was shown/measured with the converter energized and the converter off but with the gate drives active. This clearly shows that significant EMI is produced by the gate drives and coupled to the supply (busbars).

When compared to a typical set-up that is used to measure EMI for regulatory purposes, the non-energized converter can produce noise that is in excess of the regulatory limit.

This study shows to the fact that designers can under estimate converter EMI that is generated by only considering noise from the modulated power conversion process and ignoring the effects of the gate drives.

\section{PARASITIC COMPONENTS}

Fig. 3, taken from [6], shows a model used for describing switching behavior of a MOSFET. It is important to note that this model is only valid for the MOSFET in the saturation region. This is a valid assumption as power electronic semiconductors in a converter is used as a switch which is either on or off-i.e. in the saturation region. The model is constructed by expressing the physical structure and semiconductor processes as circuit components [6]. For example the inductances model the connections for the drain $(D)$ and source $(S)$ where the connection is in a loop that cuts through magnetic flux generated by the connection. The capacitances are from pads in close proximity to each other with doped semiconductor in between as dielectric. The model for the gate connection $(G)$ includes resistance due to a path through the doped

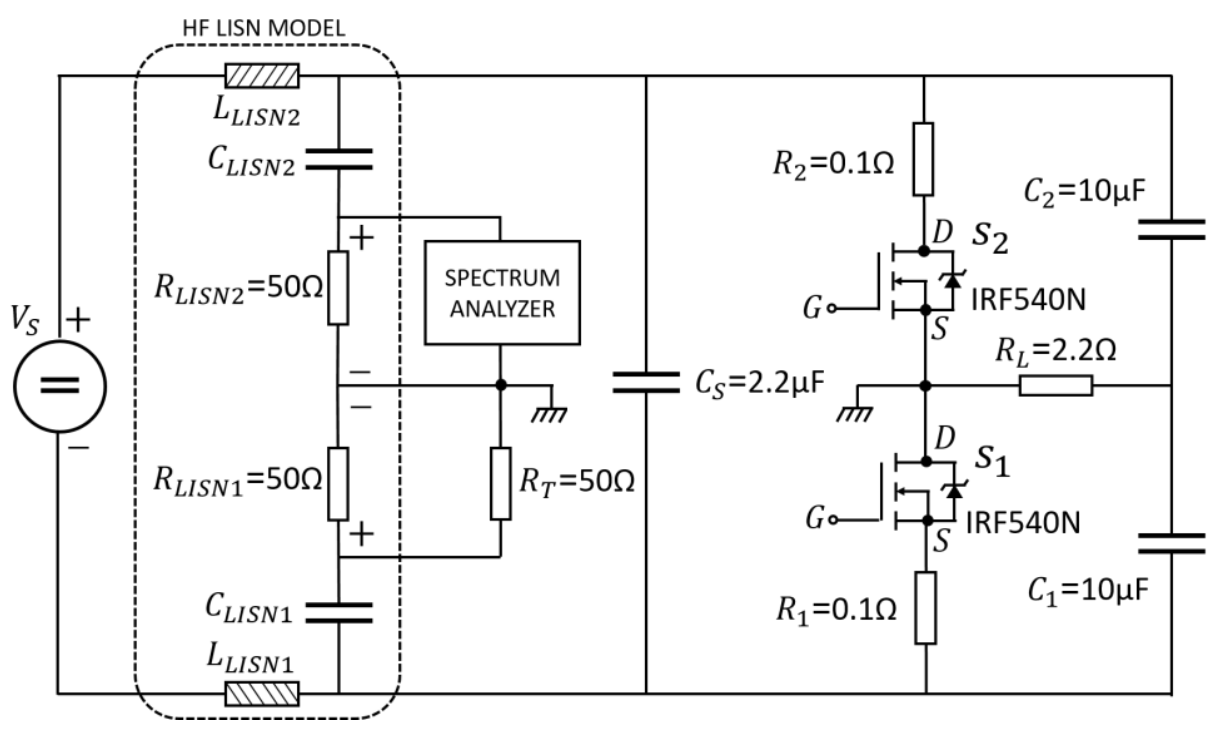

Fig. 4. Practical circuit used for measurements 


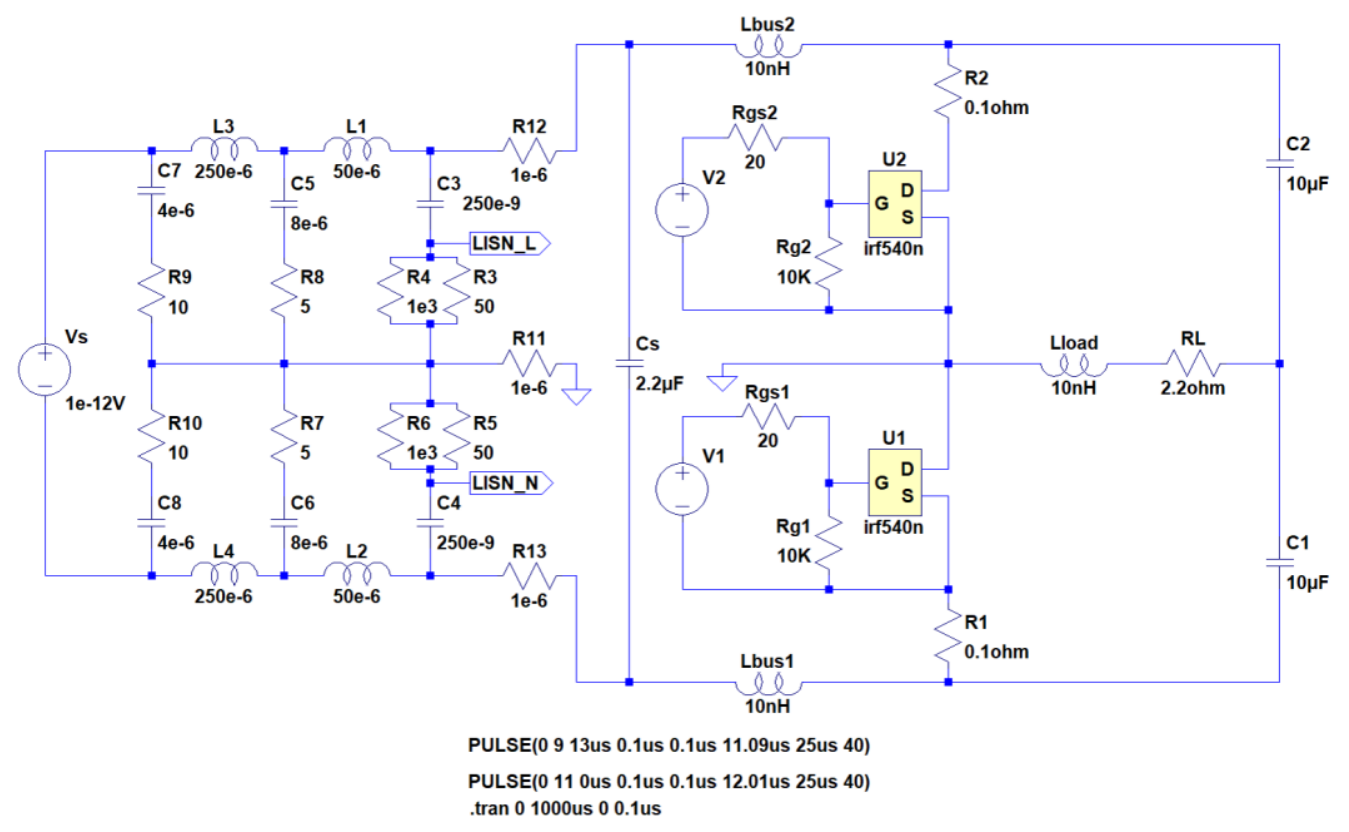

Fig. 5. SPICE simulation of the practical circuit.

semiconductor gate region.

In this study two conditions in converter action are considered. The first is when the converter is actively switching current and transferring energy from source to load. In Fig. 1 this would imply that $I_{D} \neq 0$. The second is when the converter is not transferring energy from source to load and $I_{D}=0$. Although the gate capacitances vary under different operating conditions the gate stays connected to the source and drain through $C_{G D}$ and $C_{G S}$ (Fig. 3). Since the gate $(G)$ is switching and connected through the parasitic capacitances, noise current will flow at $I_{D C}$ in Fig. 1.

\section{PRactical Circuit}

The practical circuit used for measurements is shown in Fig. 4. It consists of a single phase arm $\left(S_{1}\right.$ and $\left.S_{2}\right)$ connected in an H-bridge fashion to two large capacitances and a load in between. Not shown are the gate drives that connect between the gate $(G)$ and source $(S)$ for each of the IRF540N MOSFETs.

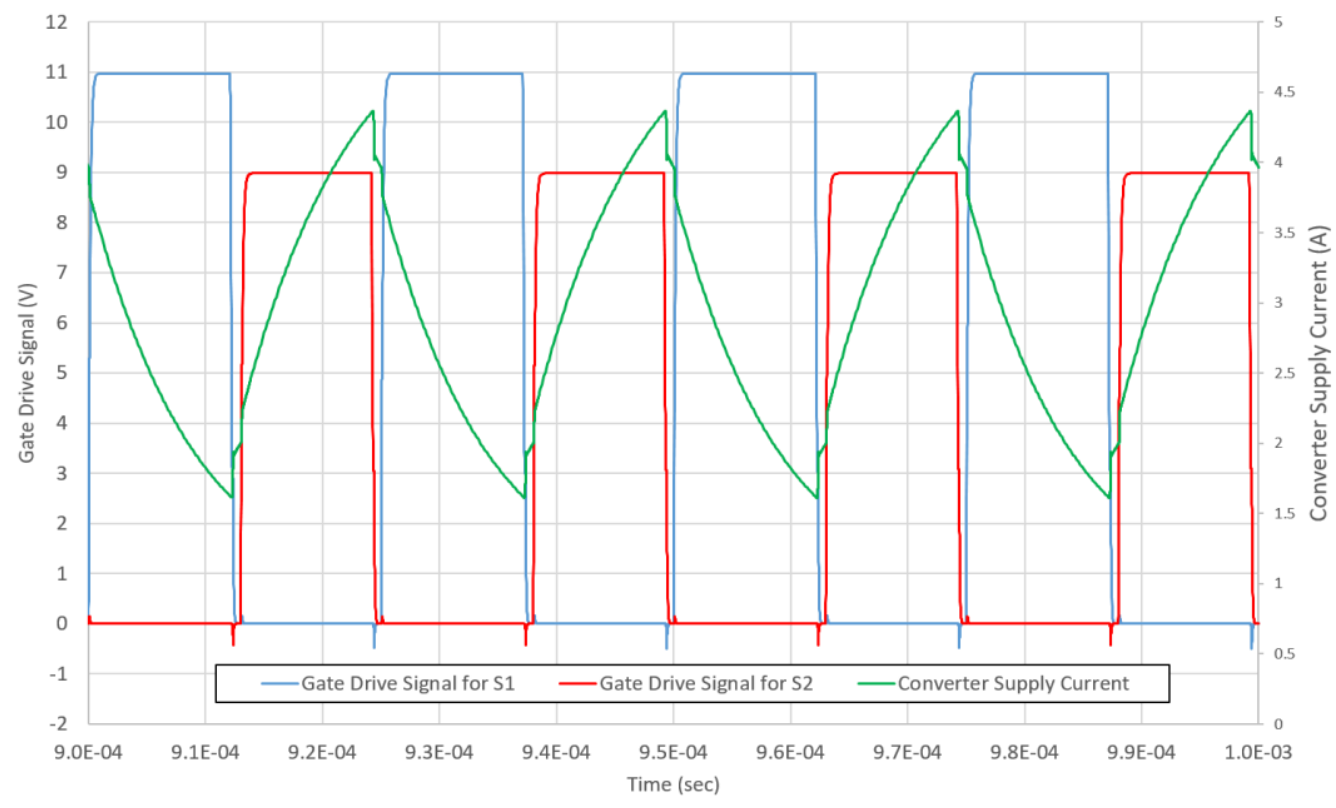

Fig. 6. SPICE simulation waveforms showing gate drive signals for S1 and S2 as well as the converter supply current. 


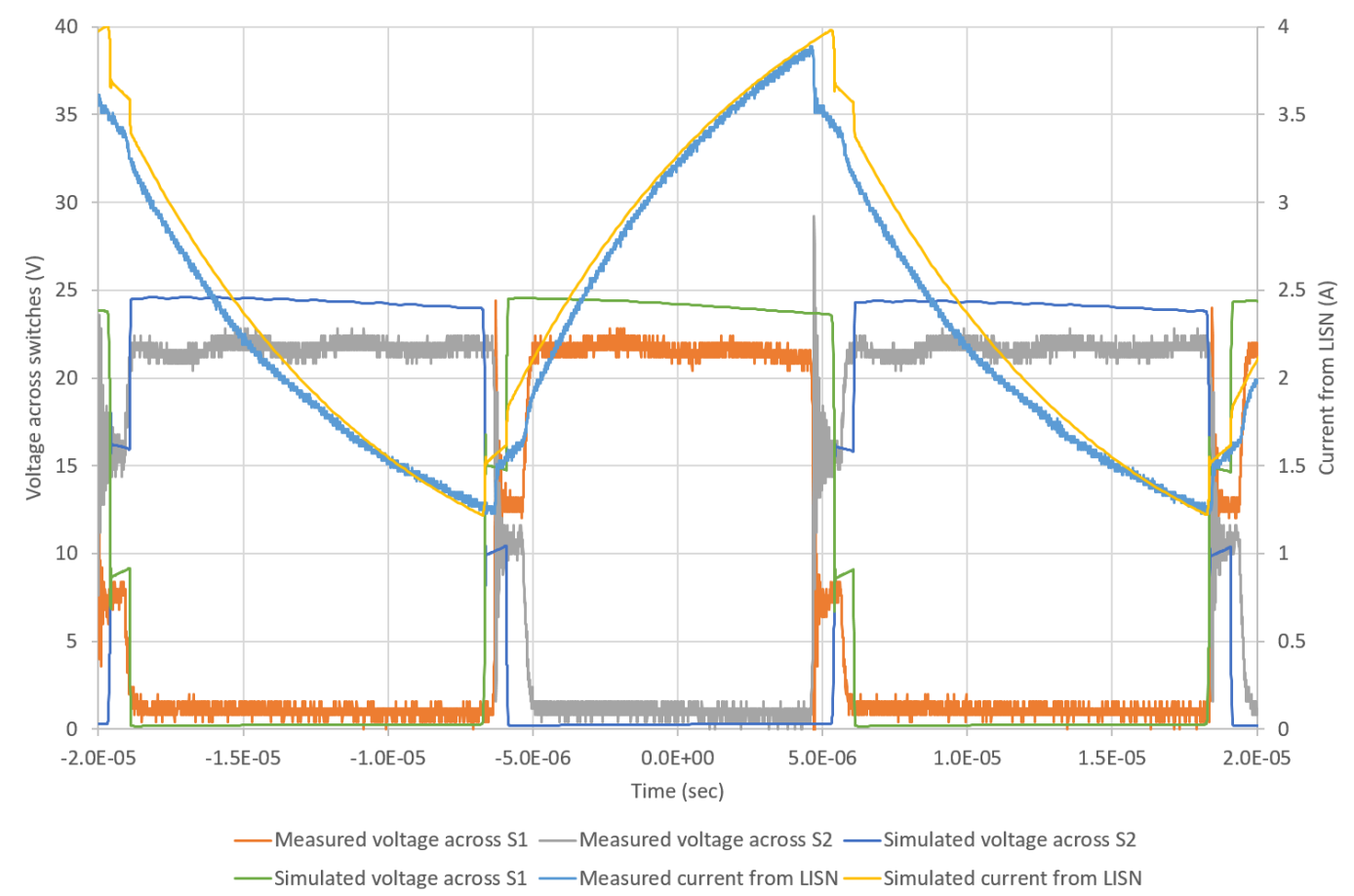

Fig. 7. Simulated waveforms and measured results for the voltages across the MOSFETs and current from the LISN for 25VDC on the busbars.

The gate drives use an IR2153 self-oscillating half-bridge driver with isolated outputs connected to the MOSFET gates.

The phase arm is fed with a DC voltage source through a Line Impedance Stabilization Network (LISN). LISN's are networks used to measure EMI on power lines during regulation testing of products. The LISN has the following features:

- It lets through the DC supply voltage for powering the circuit that is being tested.

- It prevent noise on the DC supply from interfering with noise measurements.

- It provides a standard $50 \Omega$ impedance (at higher frequencies - typically above $1 \mathrm{MHz}$ ) to the noise source.

- It outputs a voltage into a $50 \Omega$ device such as a spectrum analyzer corresponding with the noise/EMI generated by the tested circuit.

The LISN shunts the EMI current. In a real life scenario, no LISN is present and all the noise current will flow through to the source.

\section{Simulation}

A simulation of the circuit in Fig. 4 was performed using SPICE. This is shown in Fig. 5 and was done to investigate converter noise behavior without having to build a physical circuit. Comparison against the measurements from the physical circuit is given under the section "Results".

The IRF540N devices were modelled using a subcircuit as provided by the manufacturer [7].

A complete LISN was modelled as to include the low frequency characteristics of the LISN.

Parasitic inductances were added to model lay-out surface area. Gate resistors were added to the simulation to mimic the practical gate waveforms.

Fig. 6 shows simulation waveforms for the gate drive signals of $S_{1}$ and $S_{2}$ as well as converter supply current for a 25VDC supply input ( $I_{D C}$ in Fig. 1). The gate drive signals differ in amplitude as it mimics the practical IR2153 circuit output. The dead time is clearly visible. The supply current is roughly triangular as would be expected from a half bridge charging capacitors $\left(C_{1}\right.$ and $\left.C_{2}\right)$ through the load resistance. The switching frequency is $40 \mathrm{kHz}$. Comparison to measurements from the practical circuit is shown in the next section.

\section{RESULTS}

In this section results for two different circuit configurations are given:

- First are the results for a circuit with an input of 25VDC on the busbars.

- $\quad$ Second are the results for a circuit with zero input on the busbars but with the gate drives live.

\section{A. Energized Busbars}

Fig. 7 shows simulated waveforms and measured results for the voltages across the MOSFETs and current from the LISN for 25VDC on the busbars. The switching waveforms were recorded to verify that operation with energized busbars was correctly modelled. The current from the LISN is $I_{D C}$ in Fig. 1 and carries the EMI. It is a version of this current that is measured with the LISN.

The IR2153 self-oscillating half-bridge driver connected to the MOSFET gates did not provide an exact $50 \%$ duty cycle and therefore the simulated waveforms are only approximately similar to the measured results. 


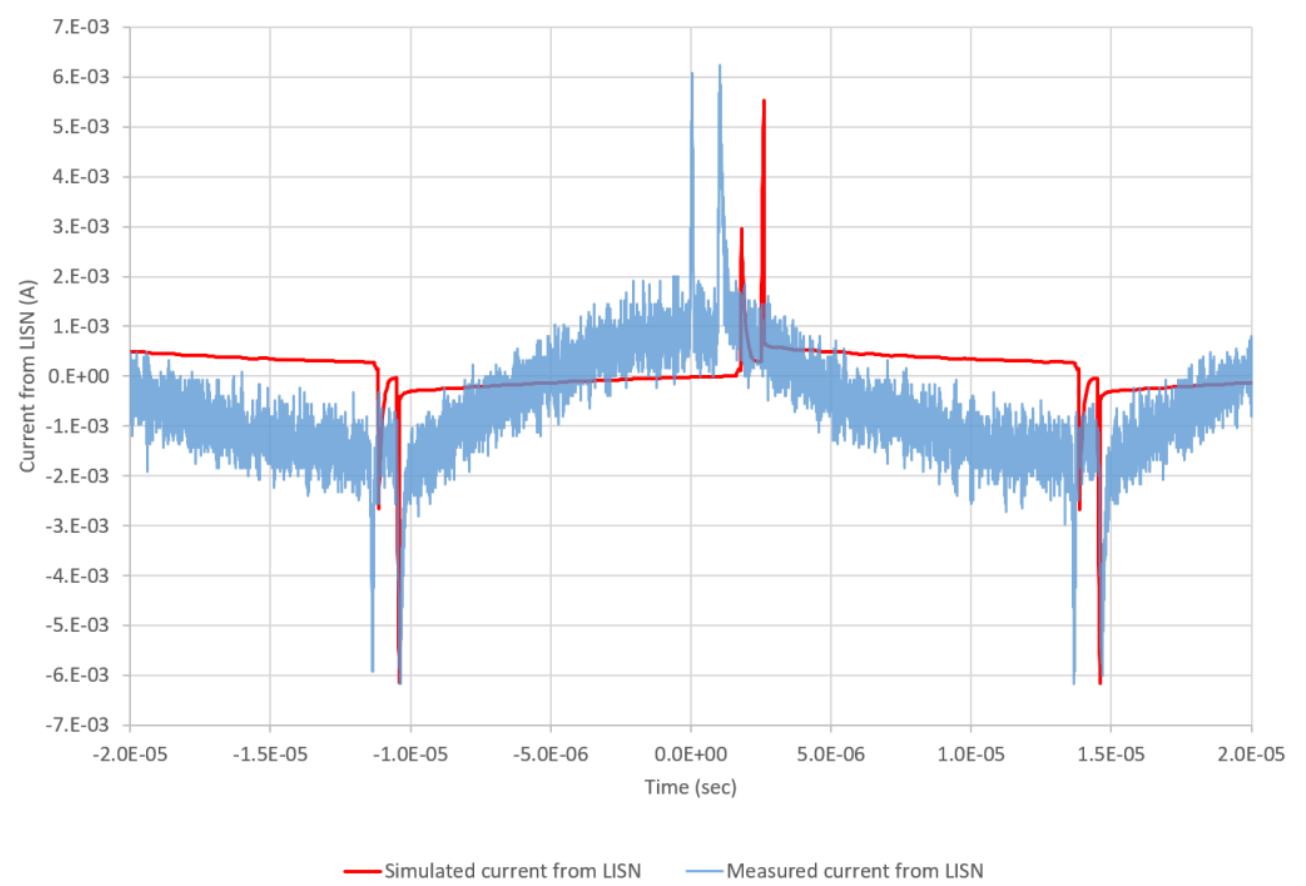

Fig. 8. Simulated waveforms and measured results for current from the LISN for no voltage applied to the busbar (non-energized).

Although having the same wave shape, the amplitude of the measured voltages across the semiconductor switches are smaller than that of the simulation. This is probably due to the fact that not all the losses in the converter were incorporated into the simulation.

There is a fair correlation between the measured supply current (or current from the LISN) and the simulated values.

The measured waveforms exhibit much more noise and overshoot than the simulated results. This is due to the fact that not all the parasitics of the converter were modelled.

From the results in Fig. 7 it is clear that the converter operation SPICE model with energized busbars is valid as it is modelled with a fair correlation to the measured behavior.

\section{B. Non-energized Busbars}

The current from the LISN is $I_{D C}$ in Fig. 1 and carries the EMI. It is a version of this current that is measured with the LISN. Fig. 8 shows this current for non-energized busbars.

Fig. 8 clearly shows the discrepancy in duty cycle between the measured and simulated currents with the positive peaks not correlating.

In comparison to the current measured in Fig. 7, the current in Fig. 8 is orders of magnitude smaller. All the energy contained in this current waveform are from the gate drives coupled through internal MOSFET capacitive parasitics.

From the discrepancy between the measured and simulated results it is postulated that the MOSFET SPICE model is not accurate in this operating region or the parasitics of the converter have a larger influence under low current conditions.

The current from Fig. 7 has a DC-offset of roughly 2.5 A. The current from Fig. 8 it is much closer to $0 \mathrm{~A}$. Therefore, from an energy conversion point of view, the energized converter will transfer energy while the non-energized converter will not. However, from an EMI point of view there is a lot of high frequency content present in the current from Fig. 8. This is in spite of the fact that this current transfers almost zero net energy from source to load. The next sub-section explores the frequency content of the energized vs. non-energized current.

\section{EMI and Spectral Content}

$I_{D C}$ in Fig. 1 is the load current carrying the energy transferred by the converter but also the EMI. In order to measure the EMI, a LISN is used [8]. The LISN measures the EMI as a high frequency content (typically $>100 \mathrm{kHz}$ ) voltage across an impedance of mainly $50 \Omega$. The LISN output spectra for the two cases of an energized vs. non-energised converter are given Fig. 9.

The spectra clearly show the switching frequency at $40 \mathrm{kHz}$ and all subsequent harmonics at multiples of $40 \mathrm{kHz}$.

For reference, the conducted emission limit from IEC 610006-3-2020 [9] is also plotted. The spectra of the two currents do not strictly comply with the IEC standard and is for rough comparison only. The measurement bandwidths differ and the limit is for a Quasi-Peak (QP) detector, while the current spectra are characterized by their peak components.

What is however significant is that the LISN spectrum for the non-energized case exceeds or is comparable to the 61000-6-3 limit. This emphasizes the main point of this paper that even if a converter has no load (non-energized) the action of the gate signals will cause significant EMI. This is due to leakage of high frequency noise through the parasitic capacitances of the semiconductor switching elements. 


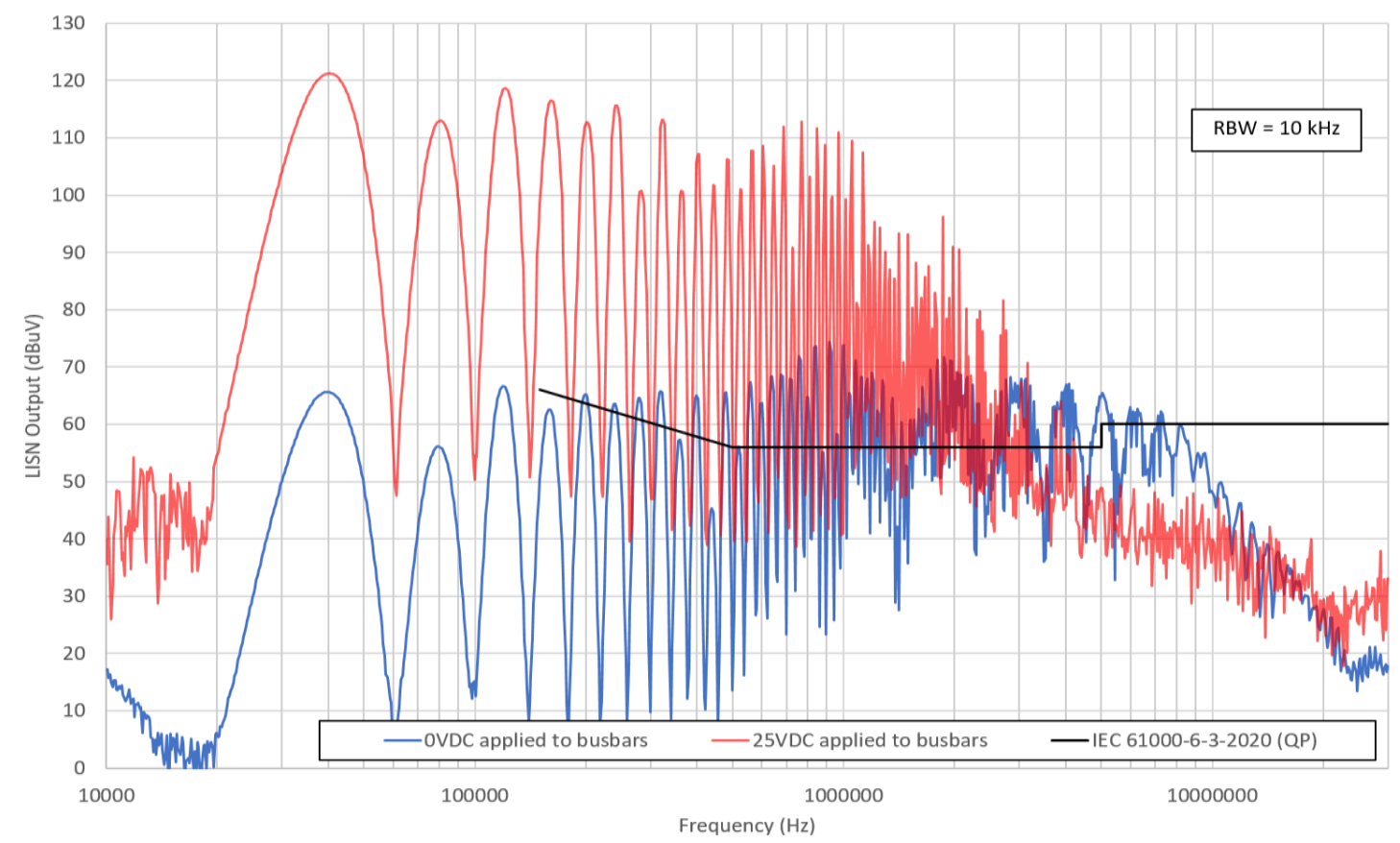

Fig. 9. Measured spectra for current from the LISN for no voltage applied to the busbar (non-energized) vs. 25VDC applied (energized).

\section{CONCLUSION}

This study shows that gate drives can have a significant influence on the EMI generated by power converters. This is mainly due to parasitic capacitances connecting the gates of the semiconductor power switches to the rest of the converter.

Even if the converter is de-energized and does not convert energy, the EMI from the gate drives can exceed statutory limits.

MOSFETs were used to construct and measure a practical half bridge converter. SPICE simulations were used to gain insight into the practical results. With the converter energized, the measured waveforms correlates well with the simulation. However, when de-energized and only the gate drives operational the simulation differs from the measured results. This is possibly due to the fact that the MOSFET SPICE model is constructed to accurately simulate large signal behavior and not behavior in the absence of drain current.

The converter described in this text - when fully energized, exceeds the IEC 61000-6-3-2020 conducted emission limits on the power line by 50 to $60 \mathrm{~dB} \mu \mathrm{V}$ as measured through a LISN. Non-energized and with the gate drive circuitry operational the limit is exceed by up to $20 \mathrm{~dB} \mu \mathrm{V}$.

The results of this paper are significant for designers of EMI mitigation techniques such as the designing of online filters or the screening of power converters. Especially in low power converters, the contribution of the gate drive noise can be a significant portion of the total EMI.

\section{REFERENCES}

[1] M. M. Biswas, M. S. Azim, T. K. Saha, U. Zobayer, and M. C. Urmi, "Towards Implementation of Smart Grid: An Updated Review on Electrical Energy Storage Systems," vol. 2013, no. 01, pp. 122-132, Feb. 2013.

[2] J. Ding, M. Nemati, C. Ranaweera, and J. Choi, "IoT connectivity technologies and applications: A survey," IEEE Access, vol. 8, pp. 67646-67673, 2020.

[3] A. Di Napoli and A. Ndokaj, "EMC and safety in vehicle drives," in Proceedings of the 2011 14th European Conference on Power Electronics and Applications, 2011, pp. 1-8.

[4] M. J. Nave, Power line filter design for switched-mode power supplies. New York: Van Nostrand Reinhold, 1991.

[5] J. Mueller, R. Thoma, E. Demircan, C. Bernicot, and A. Juge, "Modeling of MOSFET parasitic capacitances, and their impact on circuit performance," Solid. State. Electron., vol. 51, no. 11-12, pp. 1485-1493, Nov. 2007.

[6] V. Barkhordarian, "Power MOSFET basics," Powerconversion Intell. Motion, vol. 22, no. 6, pp. 28-39, 1996.

[7] "IRF540N - Infineon Technologies." [Online]. Available: https://www.infineon.com/cms/en/product/power/mosfet/12v-300vn-channel-power-mosfet/irf540n/. [Accessed: 08-Jul-2021].

[8] A. S. De Beer, G. N. Wooding, and J. D. Van Wyk, "Problematic aspects when using a LISN for converter EMI characterisation," in 2013 IEEE International Conference on Industrial Technology (ICIT), 2013, pp. 633-637.

[9] IEC, "IEC 61000 - Electromagnetic compatibility (EMC)- Part 6-3 Generic standards - Emission standard for equipment in residential environments," Int. Electrotech. Comm., vol. 2004, pp. 1-13, 2020. 


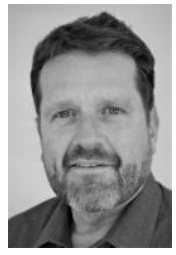

Arnoldus S de Beer was born in South Africa and received the B.Ing and M.Ing degrees in electrical engineering from the Rand Afrikaans University, Johannesburg, South Africa, in 1989 and 1992. In 1995 he was awarded the D.Ing (Electrical) degree from the Rand Afrikaans University, Johannesburg, South Africa.

Currently, Dr de Beer lectures at the University of Johannesburg, Johannesburg, South Africa since 2007.

Previously, he was with various commercial companies in South Africa.

His research interests include Electromagnetic Compatibility (EMC) in power electronics, power-line communications and EMC in the smart-grid and Internet of Things (IoT). 